\title{
Malignant Mixed Tumor of the Minor Salivary Gland
}

National Cancer Institute

\section{Source}

National Cancer Institute. Malignant Mixed Tumor of the Minor Salivary Gland. NCI

Thesaurus. Code C5955.

A malignant tumor arising from the minor salivary glands. It includes carcinoma ex pleomorphic adenoma, a malignant epithelial tumor arising from a pre-existing pleomorphic adenoma and carcinosarcoma which is characterized by a mixture of malignant epithelial and sarcomatous elements. 